\section{Síndrome de Cushing iatrogénico y sarna costrosa por uso de esteroides tópicos}

Edna Morán-Villaseñor, ${ }^{1}$ Carolina Palacios-López, ${ }^{2}$ Marimar Sáez de Ocariz, ${ }^{2}$ Luz Orozco-Covarrubias, ${ }^{2}$ Carola Durán-McKinster ${ }^{3}$

\begin{abstract}
Resumen
El uso prolongado de esteroides tópicos en niños se puede asociar con efectos adversos locales y sistémicos. Se presenta el caso de una paciente de seis meses de edad con síndrome de Cushing iatrogénico y sarna costrosa tras el uso de betametasona al $0.05 \%$ en el área del pañal. La sarna costrosa es una variedad de escabiosis que tiene la particularidad de ser más extensa y más contagiosa; generalmente se observa en pacientes inmunodeprimidos. En este caso fue favorecida por la aplicación crónica de esteroides tópicos.

Los médicos de primer contacto y los padres deben conocer los efectos secundarios potenciales de los esteroides tópicos, especialmente en áreas de piel delgada o cubierta (como el área del pañal) en donde la absorción de los esteroides tópicos se incrementa.
\end{abstract}

PALABRAS CLAVE: síndrome de Cushing iatrogénico, sarna costrosa, esteroides tópicos.

Acta Pediatr Mex. 2018 Mar;39(2):172-178.

\section{latrogenic Cushing's syndrome and crusted scabies due to misuse of topical steroid therapy.}

Edna Morán-Villaseñor, ${ }^{1}$ Carolina Palacios-López, ${ }^{2}$ Marimar Sáez de Ocariz, ${ }^{2}$ Luz Orozco-Covarrubias, ${ }^{2}$ Carola Durán-McKinster ${ }^{3}$

\begin{abstract}
Prolonged use of topical corticosteroids in children may be associated with both local and systemic adverse effects. We report a case of a 6-month-old female who developed Cushing's syndrome and crusted scabies due to misuse of betamethasone in the diaper area for three months. Crusted scabies is a form of scabies that has the particularity of being more extensive and highly contagious, it is most commonly observed in immunosuppressed patients, in this case it was induced by the chronic application of topical corticosteroids.
\end{abstract}

Physicians and parents should be aware of the potential side effects of topical corticosteroids, especially in thin and covered skin areas, like the diaper area, where absorption is increased.

KEYWORDS: iatrogenic Cushing's syndrome; crusted scabies; topical corticosteroids

\footnotetext{
${ }^{1}$ Residente de Dermatología Pediátrica.

${ }^{2}$ Adscrita al servicio de Dermatología.

${ }^{3}$ Jefa del servicio de Dermatología.
}

Instituto Nacional de Pediatría, México.

Recibido: 30 de septiembre 2016

Aceptado: 19 de abril 2017

Correspondencia

Carolina Palacios López

caroderma@yahoo.com.mx

Este artículo debe citarse como

Morán-Villaseñor E, Palacios-López C, Sáez-deOcariz M, Orozco-Covarrubias L, Durán-McKinster C. Síndrome de Cushing iatrogénico y sarna costrosa por uso de esteroides tópicos. Acta Pediatr Mex. 2018;39(2):172-178. 


\section{INTRODUCCIÓN}

Desde su aparición, en 1952, los esteroides tópicos representan uno de los principales pilares en el tratamiento de muchas enfermedades dermatológicas gracias a su efecto antiinflamatorio y antiproliferativo. Si se aplican de forma adecuada son seguros y eficaces; sin embargo, sin una supervisión médica correcta pueden producir graves efectos adeversos locales y sistémicos. ${ }^{1}$ Los niños, en particular los lactantes, son más propensos a manifestar estos efectos adversos, por lo que se debe vigilar estrechamente su uso en población pediátrica.

Un efecto adverso sistémico grave es el síndrome de Cushing iatrogénico que, aunque es poco frecuente, repercute de forma importante en la salud del paciente. El uso de esteroides tópicos de alta potencia también favorece la aparición de infecciones cutáneas o las empeora.

Se presenta el caso de una paciente de seis meses de edad con síndrome de Cushing iatrogénico y sarna costrosa como efectos adversos al uso de una crema con betametasona tópica, al 0.05\%, combinada con clotrimazol y gentamicina.

\section{PRESENTACIÓN DE CASO CLÍNICO}

Niña de seis meses de edad, previamente sana, sin antecedentes heredofamiliares o personales patológicos de importancia. Fue llevada al servicio de Dermatología por una dermatosis de 5 meses de evolución. A su ingreso la exploración física reportó: peso de $4.910 \mathrm{~kg}$ (percentila $<5$, Z score -2.99) y talla de $53 \mathrm{~cm}$ (percentila $<5$, Z score -4.52), con cara de luna llena, mejillas prominentes, hipertricosis de predominio en frente, mejillas y mentón; así como giba dorsal (Figura 1).

Se observó dermatosis diseminada al tronco y las extremidades; en el tronco predominaba en el pecho, abdomen y genitales y en las extremi-

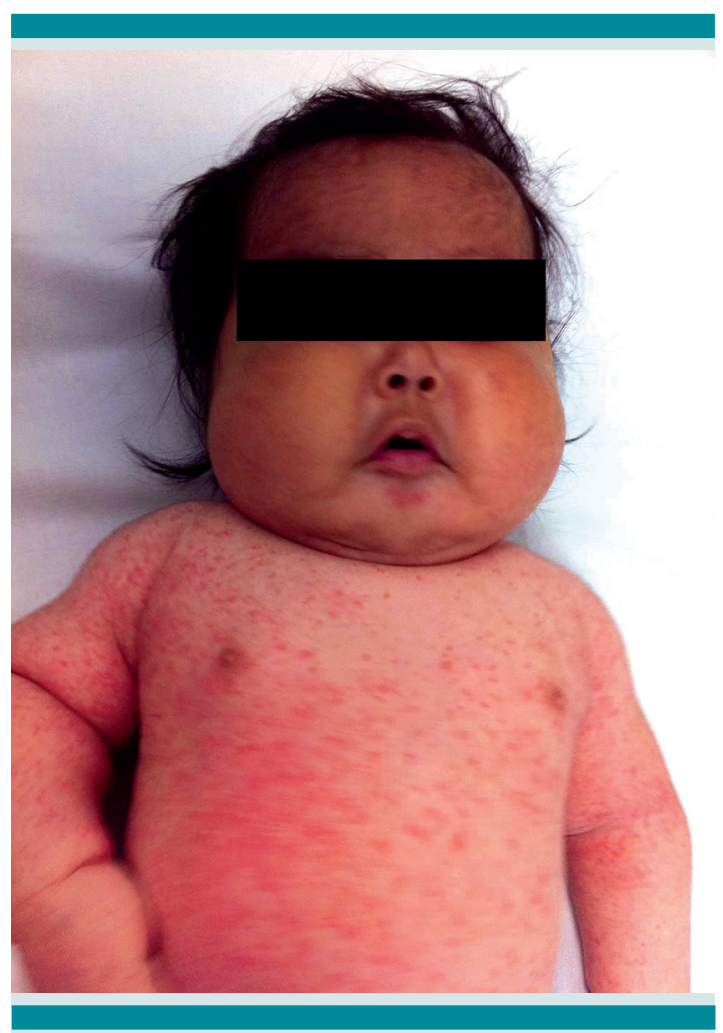

Figura 1. Paciente de 6 meses de edad con cara de luna Ilena, mejillas prominentes e hipertricosis en la frente, mejillas y mentón.

dades predominaba en las palmas y las plantas; estaba constituida por múltiples pápulas eritematosas, milimétricas, pruriginosas y algunas costras hemáticas (Figura 2), así como placas queratósicas costrosas (en palmas y plantas) y paquioniquia en las uñas de las manos y pies (Figura 3).

La madre informó que desde la tercera semana de vida se le aplicó dipropionato de betametasona al $0.05 \%$, clotrimazol y gentamicina en crema, cada tercer día, por una supuesta dermatitis en el área del pañal, con una dosis acumulada total de esteroide de 4.28 gramos tópico en base a hidrocortisona. A los 3 meses de edad le aparecieron las lesiones costrosas descritas y la madre notó la detención del crecimiento (sin incrementos en el peso ni en la talla). 


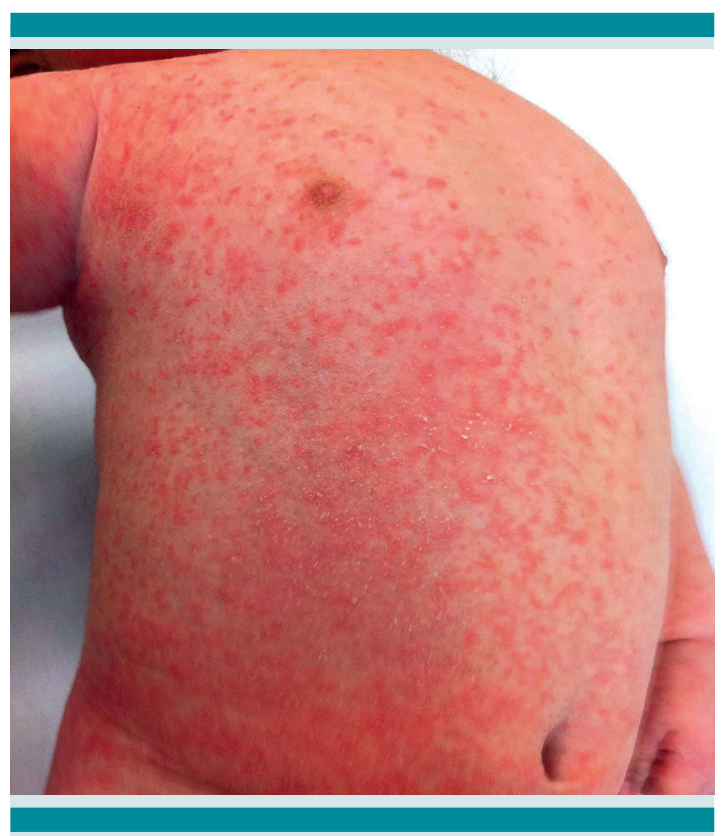

Figura 2. Múltiples pápulas eritematosas, milimétricas y algunas costras hemáticas de predominio en el tronco y las extremidades.

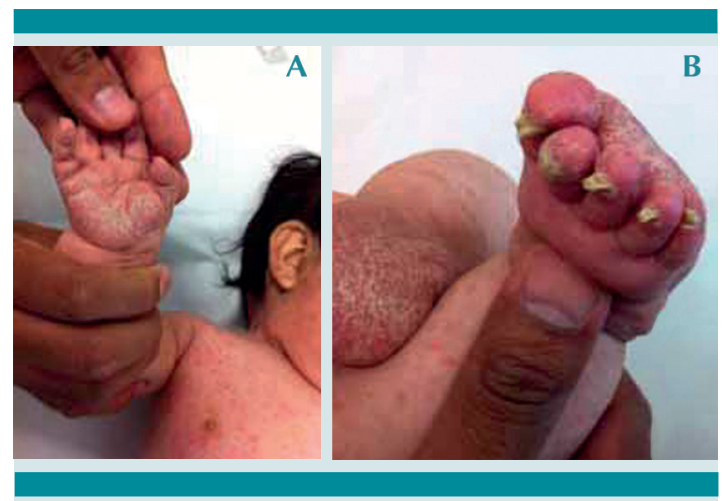

Figura 3. A) Placas queratósicas en las palmas. B) Paquioniquia en las uñas de los pies.

Con base en los hallazgos clínicos se diagnosticaron síndrome de Cushing iatrogénico y sarna costrosa; y tras la revisión de sus familiares se hizo el diagnóstico de escabiosis en la madre, padre y un hermano de 2 años de edad. Se suspendió el esteroide tópico y se dio tratamiento con vaselina azufrada al 5\%. Los padres y el hermano fueron tratados con ivermectina vía oral (12 mg y $200 \mu \mathrm{g} / \mathrm{kg}$, respectivamente) en dosis única, con la indicación de repetir el tratamiento a los 15 días.

El servicio de Endocrinología le prescribió prednisona por vía oral en dosis de reducción.

Se revaloró a la paciente al mes de tratamiento y fue evidente la desaparición de las lesiones costrosas; sin embargo, persistía la facies cushingoide (Figura 4). Durante el seguimiento se evidenció la desaparición de las manifestaciones cutáneas del síndrome de Cushing iatrogénico y recuperación del peso y la talla. Un año después de su primera consulta persistía con peso y talla por debajo de la percentila 5 (peso de $9.05 \mathrm{~kg}, Z$ score de 2.17, previo de -2.99 y talla de $72.6 \mathrm{~cm} Z$ score de -2.67, previo de -4.52).

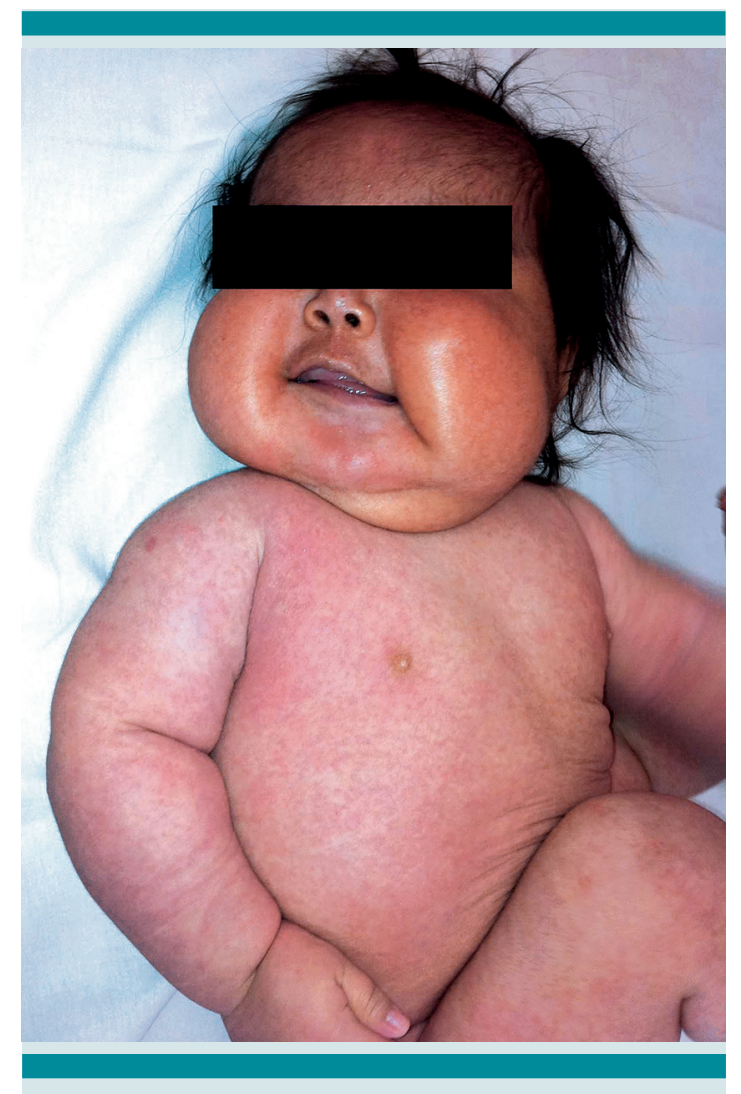

Figura 4. Aspecto de la paciente después de un tratamiento con vaselina azufrada; nótese la disminución en las lesiones de la piel; sin embargo, persiste la facies cushingoide. 


\section{DISCUSIÓN}

Los esteroides tópicos son la primera línea de tratamiento para muchas dermatosis por su variedad de efectos antiinflamatorios, inmunosupresores antiproliferativos y vasoconstrictores. ${ }^{2}$ Su uso adecuado permite que sean seguros y eficaces; sin embargo, su uso incorrecto causa efectos adversos locales y sistémicos ${ }^{1,2}$ (Cuadro 1).

Cuadro 1. Principales efectos adversos del uso de esteroides tópicos ${ }^{1,2}$

\begin{tabular}{|c|}
\hline Efectos locales \\
\hline Cambios atróficos \\
\hline Atrofia cutánea \\
\hline Telangiectasias \\
\hline Estrías \\
\hline Púrpura \\
\hline úlceras \\
\hline Infecciones \\
\hline $\begin{array}{l}\text { Enmascaramiento de infecciones cutáneas (tiña } \\
\text { incógnito) }\end{array}$ \\
\hline $\begin{array}{l}\text { Empeoramiento de infecciones cutáneas (herpes simple, } \\
\text { candidiasis, escabiosis o molusco contagioso) }\end{array}$ \\
\hline Oculares \\
\hline Glaucoma \\
\hline Cataratas \\
\hline Misceláneos \\
\hline Erupciones acneiformes \\
\hline Dermatitis perioral \\
\hline Rosácea por esteroides \\
\hline Hirsutismo \\
\hline Cambios en la pigmentación \\
\hline Retraso en la cicatrización de heridas \\
\hline Efectos sistémicos \\
\hline Supresión del eje hipotálamo-hipófisis-suprarrenal \\
\hline Síndrome de Cushing \\
\hline Hiperglucemia \\
\hline Hipocalcemia \\
\hline Hipertensión arterial sistémica \\
\hline Osteopatía \\
\hline
\end{tabular}

Modificado de Hengge et al. ${ }^{1}$
Existen diversos factores que incrementan la absorción de los medicamentos en la piel y, por lo tanto, aumentan la probabilidad de causar efectos adversos. En nuestra paciente varios de estos factores influyeron en la aparición del síndrome de Cushing iatrogénico y de la sarna costrosa:

1. Potencia del esteroide utilizado. Los efectos adversos se pueden presentar con cualquier esteroide tópico, incluso con los de baja potencia como la hidrocortisona al $1 \%{ }^{3}$ sin embargo, son los esteroides de mayor potencia los que se asocian más frecuentemente con efectos adversos. Se ha descrito que incluso la aplicación durante pocos días de $2 \mathrm{~g}$ de propionato de clobetasol al $0.05 \%$ (esteroide clase 1 o superpotente) es suficiente para suprimir el eje hipotálamo-hipófisis-suprarrenal. ${ }^{1}$ En nuestra paciente se aplicó dipropionato de betametasona al $0.05 \%$ en crema, que es un esteroide superpotente. ${ }^{2}$

2. Sitio de aplicación del esteroide. La absorción del medicamento es menor al 1\% en zonas como las palmas o las plantas, donde la piel es más gruesa; sin embargo, en áreas de piel delgada como párpados, escroto o área del pañal la absorción puede llegar a ser del 35\%. ${ }^{4}$

3. Aplicación sobre una piel enferma que ha perdido su función de barrera (como en dermatitis atópica o dermatitis del área del pañal). ${ }^{5-7}$ Esto incrementa la absorción del fármaco 2 a 10 veces más en comparación con la absorción en piel sana. ${ }^{1}$

4. Cobertura de la zona donde se aplica el esteroide. ${ }^{5,8}$ El uso de pañal en lactantes tiene efecto oclusivo.

5. Edad del paciente. Los lactantes tienen mayor proporción de superficie corporal cutánea en relación con su peso, lo que aumenta la absorción cutánea de cualquier medicamento tópico. ${ }^{2,5}$ 
Los factores mencionados permiten entender porqué los niños son más susceptibles a los efectos adversos causados por el uso de esteroides tópicos.

En un informe de la FDA (US Food and Drug Administration) se revisaron 22 casos de insuficiencia o supresión adrenal, síndrome de Cushing iatrogénico o retraso del crecimiento secundarios al uso de esteroides tópicos, en pacientes entre 6 meses y 15 años de edad. La indicación más común para el uso del esteroide tópico fue la dermatitis atópica (7 casos) y en segundo lugar la dermatitis del área del pañal (6 casos). En 11 de los 22 casos el medicamento se utilizó durante más de 3 meses, incluyendo 7 pacientes en los que se utilizó durante más de un año. El esteroide más frecuentemente utilizado fue betametasona (en 5 casos valerato de betametasona y en 3 dipropionato de betametasona) seguido del propionato de clobetasol al $0.05 \%$ (7 casos). ${ }^{9}$ Estos datos son similares a lo ocurrido con nuestra paciente en quien se aplicó dipropionato de betametasona al 0.05\% durante más de 3 meses por una dermatitis del área del pañal.

El síndrome de Cushing es el resultado de la exposición prolongada e inapropiada a concentraciones excesivas de glucocorticoides circulantes, ya sean de producción endógena o exógena. ${ }^{10}$ La causa más frecuente en la edad pediátrica es la administración exógena o iatrogénica; es una entidad poco frecuente en niños, se han descrito cerca de 2-5 casos nuevos por millón de habitantes por año y de éstos sólo $10 \%$ ocurre en niños. ${ }^{11}$

La manifestación clínica más frecuente en pacientes pediátricos es el incremento de peso asociado a la detención en la talla. Otras manifestaciones son: cefalea, hipertensión arterial, hipertricosis, acné, estrías violáceas y acantosis nigricans. ${ }^{11}$ En nuestro caso hubo detención del crecimiento (peso $4.9 \mathrm{~kg}$ con Z score -2.99 y talla de $53 \mathrm{~cm}$ con $Z$ score -4.52 ), hipertricosis en la cara, facies de luna llena y giba dorsal. La mayoría de estas manifestaciones son reversibles con el tratamiento adecuado del síndrome de Cushing; sin embargo, el retraso en el crecimiento puede repercutir en la talla final del paciente.

Existen diversos reportes de síndrome de Cushing iatrogénico secundario al uso de una combinación de esteroide, antimicótico y antibiótico tópicos (dipropionato de betametasona, clotrimazol y gentamicina). ${ }^{9}$ Esta combinación es comúnmente utilizada en la población pediátrica mexicana por su fácil accesibilidad, por su bajo costo y por la falta de conocimiento de los efectos adversos que pueden sobrevenir con la aplicación de esteroides tópicos y sistémicos, en especial si se utilizan en áreas de piel delgada, lastimada y bajo oclusión, como la del pañal.

Es importante reconocer este síndrome para dar un tratamiento integral. Además de suspender el esteroide tópico se debe administrar un esteroide por vía oral en dosis de reducción dado que, por la administración prolongada, hay supresión del eje hipotálamo-hipófisis-suprarrenal, la suspensión abrupta del esteroide tópico o un evento agudo debe ser tratado con hidrocorticoides porque puede desencadenarse una crisis suprarrenal aguda o un cuadro de insuficiencia suprarrenal que podría ser mortal. ${ }^{6}$

Otros efectos adversos de los esteroides tópicos se deben a su capacidad inmunodepresora, la cual favorece o empeora ciertas infecciones en la piel. ${ }^{1,5}$

La escabiosis, comúnmente conocida como sarna, es una parasitosis cutánea muy contagiosa ocasionada por el ácaro Sarcoptes scabiei, variedad hominis. Se estima que afecta aproximadamente a 300 millones de personas al año a escala mundial. ${ }^{12}$ En México la incidencia es de 55 casos por cada 100,000 habitantes ${ }^{13}$ Clínicamente se caracteriza por pápulas eritematosas, vesículas, nódulos y túneles, así como costras 
hemáticas y escoriaciones debidas al intenso prurito. ${ }^{12}$ La variedad costrosa se caracteriza por placas queratósicas con grietas, fisuras y escaso prurito. $^{13}$

A diferencia de la sarna clásica, donde el promedio de ácaros en un paciente infectado es de $10 \mathrm{a}$ $15,{ }^{12}$ en la variedad costrosa hay una infestación por miles a millones de ácaros, lo que la hace mucho más contagiosa que la variedad clásica. ${ }^{14}$

La sarna costrosa generalmente ocurre en pacientes inmunocomprometidos, con antecedente de aplicación de esteroides tópicos, diabéticos, infectados por el virus de inmunodeficiencia humana, con leucemia de células T, linfomas, enfermedades autoinmunitarias o malnutrición, así como pacientes física o mentalmente discapacitados. ${ }^{12,14,15}$

En condiciones normales ocurre una reacción de hipersensibilidad mediada por células a los antígenos del ácaro, responsables de las manifestaciones clínicas y de limitar la infestación. Sin embargo, cuando se altera la inmunidad celular se favorece la infestación por miles a millones de ácaros, dando lugar a un cuadro de sarna costrosa. ${ }^{14}$

De acuerdo con las Guías de los Centros para el Control y la Prevención de Enfermedades (US Centers for Disease Control and Prevention) el tratamiento de la sarna costrosa requiere el uso de escabicidas tópicos y sistémicos. ${ }^{16}$ Dado que la ivermectina por vía oral no está aprobada para pacientes menores de 2 años o menores de $15 \mathrm{~kg}^{12}$ se decidió indicar vaselina azufrada al $5 \%$, con lo que se alivian las lesiones.

\section{CONCLUSIONES}

La aplicación incorrecta de esteroides tópicos tiene efectos adversos locales y sistémicos, espe- cialmente en lactantes. Pueden ocasionar atrofia cutánea, empeorar infecciones cutáneas, retrasar la cicatrización de heridas e incluso suprimir el eje hipotálamo-hipófisis-suprarrenal y ocasionar un síndrome de Cushing iatrogénico. Es necesario que tanto los médicos de primer contacto, como los pediatras, conozcan estos efectos al momento de prescribir esteroides tópicos en niños, especialmente en áreas de piel delgada (como el área del pañal) donde la absorción del medicamento se incrementa. Algunas estrategias para disminuir estos efectos adversos incluyen administrar esteroides de baja potencia y durante el menor tiempo posible, así como la educación a los padres acerca de la aplicación correcta de los esteroides tópicos, sobre todo en países como México donde estos medicamentos se pueden adquirir sin prescripción médica.

\section{REFERENCIAS}

1. Hengge UR, Ruzicka T, Schwartz RA, Cork MJ. Adverse effects of topical glucocorticosteroids. J Am Acad Dermatol. 2006;54(1):1-15.

2. Cheirif WO, Sáez de Ocariz MM, Lammoglia OL. Esteroides tópicos: revisión actualizada de sus indicaciones y efectos adversos en dermatología. Dermatología CMQ. 2015;13(4):305-12.

3. Halverstam CP, Vachharajani A, Bayliss MS. Cushing syndrome from percutaneous absorption of $1 \%$ hydrocortisone ointment in Netherton syndrome. Pediatr Dermatol. 2007;24(1):42-5.

4. Feldmann RJ, Maibach HI. Regional variation in percutaneous penetration of $14 \mathrm{C}$ cortisol in man. J Invest Dermatol. 1967;48(2):181-3.

5. Semiz S, Isik BY, Ergin S, Candemir M, Polat A. Two cases of Cushing's syndrome due to overuse of topical steroid in the diaper area. Pediatr Dermatol. 2008;25(5):544-7.

6. Siklar Z, Bostanci I, Atli Ö, Dallar Y. An infantile Cushing syndrome due to misuse of topical steroid. Pediatr Dermatol. 2004;21(5):561-3.

7. Ho CWL, Loke KY, Lim YYJ, Lee YS. Exogenous Cushing syndrome: A lesson of diaper rash cream. Horm Res Paediatr. 2014;82(6):415-8.

8. Nieman LK. Consequences of systemic absorption of topical glucocorticoids. J Am Acad Dermatol. 2011;65(1):250-2.

9. Karwoski CB. Postmarketing Safety Review - PID D030565. Drugs: Topical Corticoesteroids. FDA Web site; 2003 September 23. Disponible en: www.fda.gov/ohrms/dockets/ac/03/ briefing/3999b1_22_karwoski-memo\%2009-26-03.doc. 
10. Newell-Price J, Bertagana X, Grossman AB, Nieman LK. Cushing's syndrome. Lancet. 2006;367:1605-17.

11. Stratakis CA. Cushing syndrome in Pediatrics. Endocrinol Metab Clin N Am. 2012;41(4):793-803.

12. Banerji A, Canadian Paediatric Society, First Nations, Inuit and Métis Health Committee. Scabies. Paediatr Child Health. 2015:20(7):395-402.

13. Plascencia GA, Proy TH, Eljure LN, Atoche DC, Calderón RC. Escabiosis: una revisión. Dermatología CMQ. 2013;11(3):217-23.
14. Gladstone HB, Darmstadt GL. Crusted scabies in an immunocompetent child: Treatment with Ivermectin. Pediatr Dermatol. 2000;17(2):144-8.

15. Tirado-Sánchez A, Bonifaz A, Montes de Oca-Sánchez G, Araiza-Santibañez J, Ponce-Olivera RM. Escabiosis costrosa en pacientes con infección por VIH/SIDA. Reporte de 15 casos. Rev Med Inst Mex Seguro Soc. 2016;54(3):397-400.

16. Workoswki KA, Bolan GA; Centers for Disease Control and Prevention. Sexally transmitted diseases treatment guidelines, 2015. MMWR Recomm Rep. 2015;64(RR-03):1-137. 